\title{
Ruptured Distal Posterior Inferior Cerebellar Artery Aneurysm Associ- ated with an Arteriovenous Malformation Detected by Intraoperative In- docyanine Green Video angiography: Case Report and Literature Review
}

\author{
Mari Kusumi ${ }^{1 *}$, Hidehiro Oka ${ }^{1}$, Hamidreza Aliabadi ${ }^{2}$, Koji Kondo ${ }^{1}$, Toshihiro Kumabe ${ }^{3}$ \\ ${ }^{1}$ Department of Neurosurgery, Kitasato University Medical Center, Kitamoto, Japan \\ ${ }^{2}$ Department of Spine and Neurosurgery Associates, California, USA \\ ${ }^{3}$ Department of Neurosurgery, Kitasato University School of Medicine, Sagamihara, Japan
}

"Corresponding author: Mari Kusumi, Department of Neurosurgery, Kitasato University Medical Center, 100-6 Arai Kitamoto, Saitama 364-8501, Japan. Tel: +81485931212; Fax:+81427788855; Email: kusumi@med.kitasato-u.ac.jp

Citation: Kusumi M, Oka H, Aliabadi H, Kondo K, Kumabe T (2018) Ruptured Distal Posterior Inferior Cerebellar Artery Aneurysm Associated with an Arteriovenous Malformation Detected by Intraoperative Indocyanine Green Video angiography: Case Report and Literature Review. J Surg: JSUR-1142. DOI: 10.29011/2575-9760. 001142

Received Date: 08 June, 2018; Accepted Date: 11 June, 2018; Published Date: 15 June, 2018

\begin{abstract}
Background: Distal Posterior Inferior Cerebellar (PICA) aneurysms are quite rare. The infrequency of distal PICA aneurysms has limited our understanding of their underlying pathology, natural history, and clinical management. Moreover, vascular anomalies are frequently noted in association with distal PICA aneurysms.

Case Description: We present a very unique case of a ruptured distal PICA aneurysm associated with an Arteriovenous Malformation (AVM) which was successfully identified by Intraoperative Indocyanin Green (ICG) video angiography. In the current era, we tend to perform Computed Tomographic Angiography (CTA) studies as the initial imaging for vascular lesions. CTA revealed the distal PICA aneurysm but failed to reveal the associated AVM. However, intraoperative ICG video angiography strongly suspected the existence of an AVM.
\end{abstract}

Conclusion: Intraoperative ICG video angiography is very useful in detecting small vessel anomalies. DSA should be considered for all distal PICA aneurysms even in the era of advanced CTA.

Keywords: Arteriovenous Malformation; Cerebral Aneurysm; Distal Posterior Inferior Cerebellar Artery; Indocyanin Green Video angiography; Intraventricular Hemorrhage

\section{Abbreviations}

$\begin{array}{lll}\text { AVM } & : & \text { Arteriovenous Malformation } \\ \text { CT } & : & \text { Computed Tomography } \\ \text { CTA } & : & \text { Computed Tomographic Angiography } \\ \text { DSA } & : & \text { Digital Subtraction Angiography } \\ \text { ICG } & : & \text { Indocyanin Green } \\ \text { IVH } & : & \text { Intraventricular Hemorrhage } \\ \text { MRI } & : & \text { Magnetic Resonance Imaging }\end{array}$
OA Occipital Artery
PICA : Posterior Inferior Cerebellar Artery
SAH : $\quad$ Subarachnoid Hemorrhage
SCA : Superior Cerebellar Artery
VA : Vertebral Artery

\section{Background}

Aneurysms arising from the Posterior Inferior Cerebral Artery (PICA) are uncommon, with a reported incidence ranging between 0.49 and $3 \%$ of all intracranial aneurysms $[1,2]$. The majority arise at the Vertebral Artery (VA)-PICA junction. Herein termed distal PICA aneurysms are a much smaller proportion, which arise from 


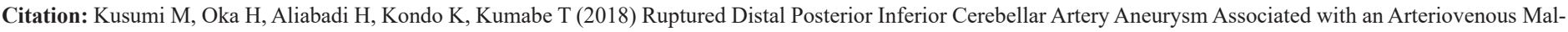

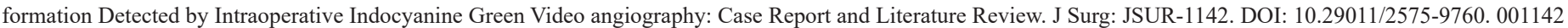

more peripheral PICA segments [2-4]. The infrequency has limited our understanding of the underlying pathology, natural history, and clinical management of distal PICA lesions [5]. In the current era of advanced non-invasive neuroimaging, we routinely perform Computed Tomographic Angiography (CTA) for detecting the cause of Subarachnoid Hemorrhage (SAH) as the initial study. Such CTA studies are often the sole examination used to detect ruptured aneurysms pre-operatively. Conventional Digital Subtraction Angiography (DSA) is often reserved for cases where the etiology of hemorrhage is unclear on non-invasive imaging or where endovascular intervention is being performed. Thus, it is often that neurovascular surgery is performed in an urgent fashion for a SAH without pre-operative DSA. However, previous reports have failed to emphasize the difficulty in diagnosing a small cerebellar Arteriovenous Malformation (AVM) associated with a distal PICA aneurysm on CTA [6].

In this paper, we report the unique case of Intraventricular Hemorrhage (IVH) with cerebellar hemorrhage caused by a ruptured distal PICA aneurysm, associated with an AVM, which was diagnosed successfully by Intraoperative Indocyanin Green (ICG) video angiography. Our case emphasizes, for distal PICA aneurysm cases, the importance of considering vascular anomalies and obtaining DSA in an era when CTAs are performed commonly and usefulness of intraoperative ICG video angiography.

\section{Case Description}

A 63-year-old man complained of sudden vertigo when straining during a bowel movement. No previous history was elicited by interview. On admission, his initial neurological examination revealed no obvious abnormalities except for vertigo, nausea, and gaze nystagmus. Computed Tomography (CT) scans revealed IVH into the fourth ventricle with a small cerebellar hemorrhage but no SAH. CTA was performed as the initial vascular imaging study and showed an aneurysm in the left distal PICA without any associated vascular anomaly (Figure 1).

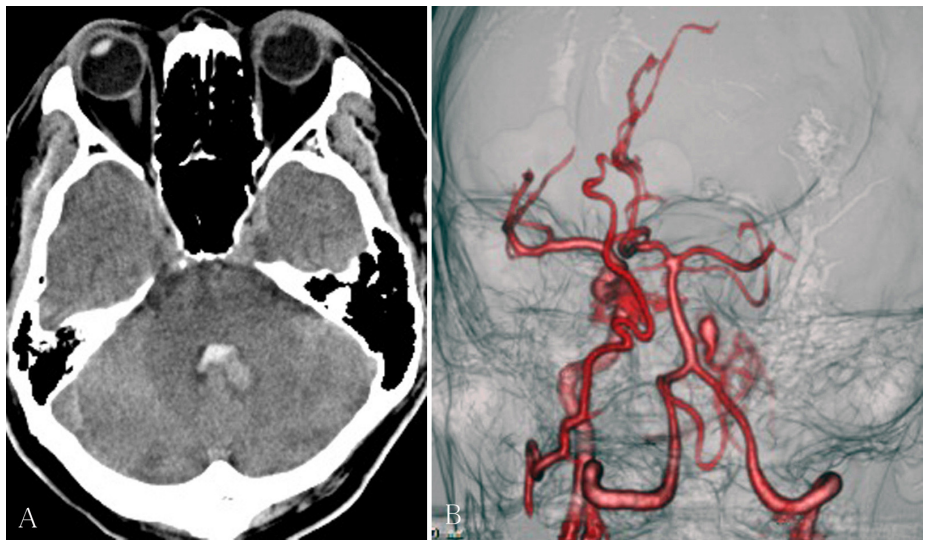

Figure 1: (A): Axial view, CT shows IVH of fourth ventricle. (B): Preoperative CTA demonstrates left distal PICA aneurysm without any other vascular anomalies.

At that point, the CTA study was felt to be adequate and thus a conventional cerebral angiogram was not performed preoperatively. The following day, we performed a suboccipital craniotomy and approached the ruptured aneurysm via the tonsillomedullary and cerebellomedullary fissures. These fissures were gently dissected and the left distal PICA branches and obex were exposed. Following sharp dissection of the fissures, the aneurysm was identified. The aneurysm however appeared to be associated with an unusual, adjacent hematoma and seemed to be connected via an abnormal vessel. Therefore, given this intraoperative finding, ICG video angiography was then performed and the aneurysm suspected to be located on a feeding point of AVM. The abnormal vessel appeared in a delayed phase, which might confirm the abnormal vessel connecting the aneurysm as feeding artery of the AVM (Figure 2). 
Citation: Kusumi M, Oka H, Aliabadi H, Kondo K, Kumabe T (2018) Ruptured Distal Posterior Inferior Cerebellar Artery Aneurysm Associated with an Arteriovenous Malformation Detected by Intraoperative Indocyanine Green Video angiography: Case Report and Literature Review. J Surg: JSUR-1142. DOI: 10.29011/2575-9760. 001142

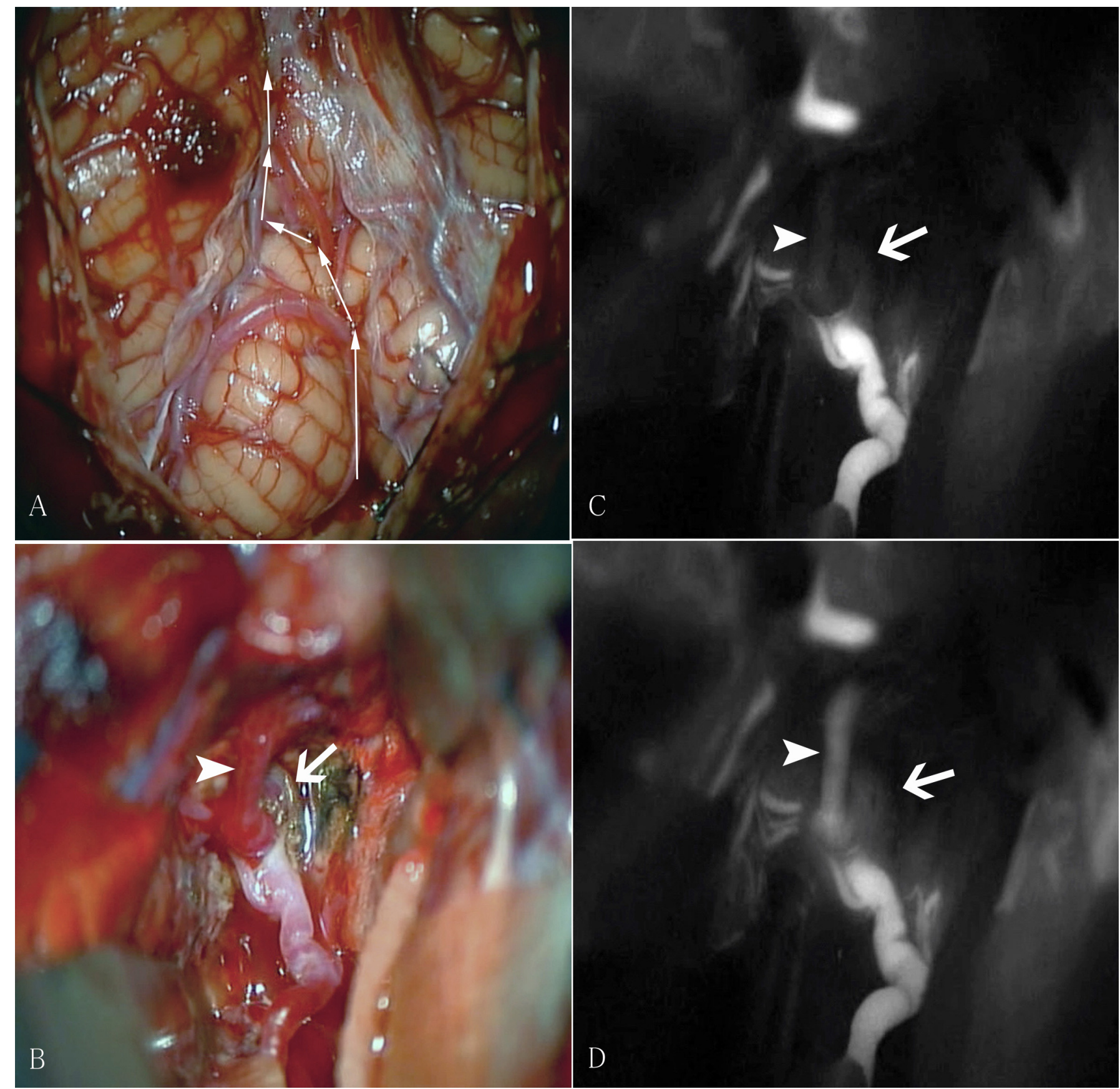

Figure 2: (A-B): Intraoperative view of mid-line suboccipital craniotomy shows normal cerebellum surface without SAH and red veins, and the view via the tonsilomedullary and cerebellomedullary fissure (A: arrow line) shows the ruptured distal PICA aneurysm (B: arrow) which appeared to be connected via an abnormal vessel (B: arrow head). (C-D): ICG video angiography (C: early phase, D: delayed phase) demonstrate delayed flow on the abnormal vessel (arrow head). The aneurysm (arrow) appeared to be located at the branching point. 


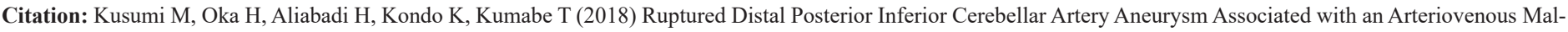

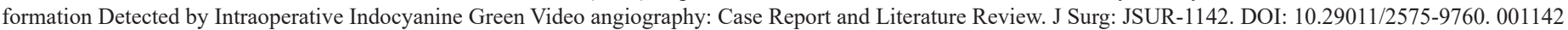

Thus, we proceeded to place a titanium mini-clip (Aesculap ${ }^{\circledR}$, Germany) just at the proximal point of the aneurysm to occlude the abnormal vessel, securing the normal branching artery of the PICA. Post-clipping ICG video angiography showed successful obliteration of the aneurysm and the abnormal vessel (Figure 3).

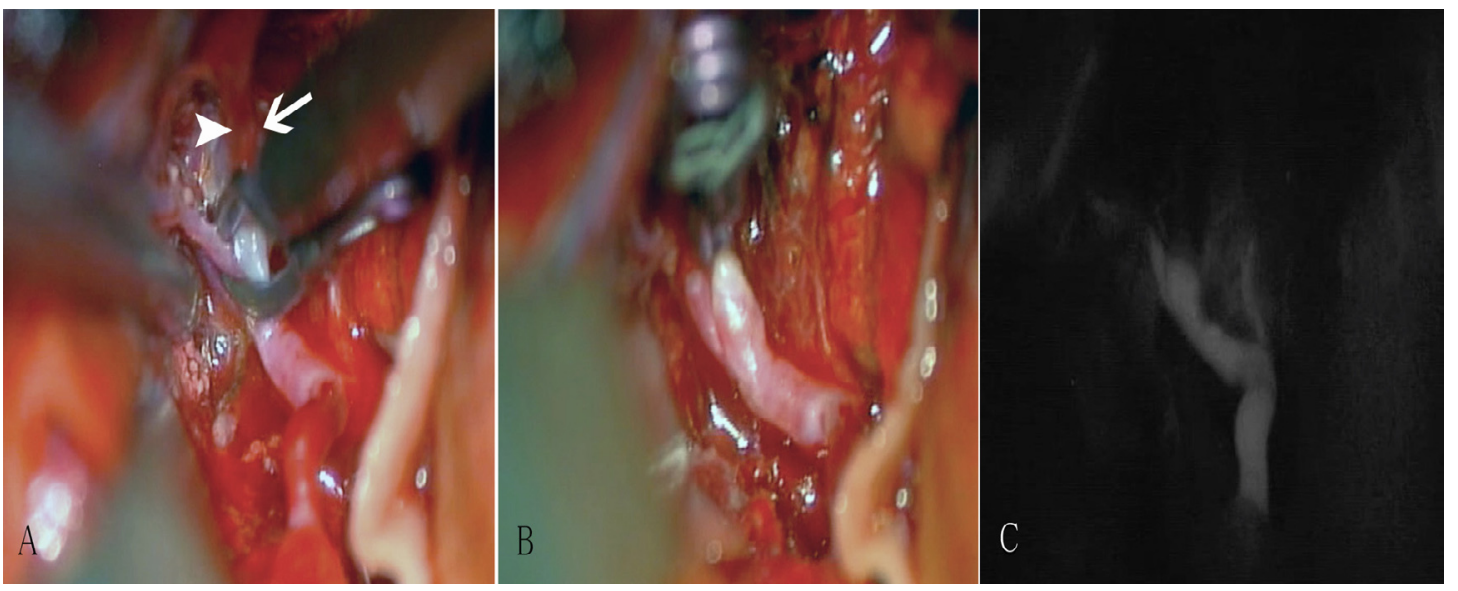

Figure 3: (A): Intraoperative view shows clip applied to the aneurysm and abnormal vessel suspected as feeding artery to AVM, with temporary clip (lower clip). (B): The next view shows final clip preserving branches of PICA. (C): ICG video angiography after clipping demonstrates disappearance of the aneurysm and abnormal vessel. Arrow: aneurysm, arrow head: abnormal vessel.

The patient's postoperative course was uneventful. Given the abnormal angioarchitecture noted during surgery, DSA was performed for further evaluation. The DSA demonstrated a superior vermian AVM fed by the left PICA and the Superior Cerebellar Artery (SCA) with complete obliteration of the aneurysm and abnormal vessel suspected as feeding artery (Figure 4). Postoperative MRI revealed no acute infarction. Stereotactic radiosurgery was scheduled for this AVM six months later, and upon completion, the AVM was completely obliterated.

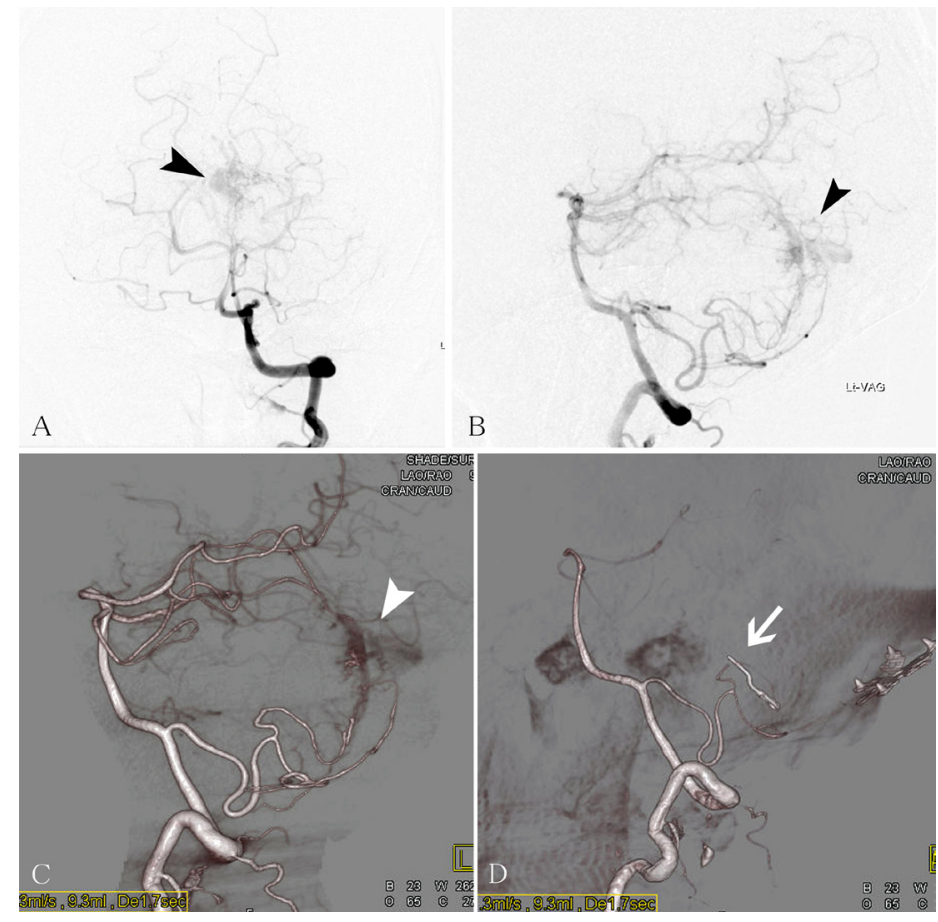

Figure 4: (A-B): Postoperative conventional DSA shows a superior vermian AVM (arrow head, A: frontal view, B: lateral view). (C-D): Three dimensional DSA demonstrates AVM fed by PICA (C: arrow head), and complete clipping of the aneurysm (D: arrow) and abnormal vessel. 


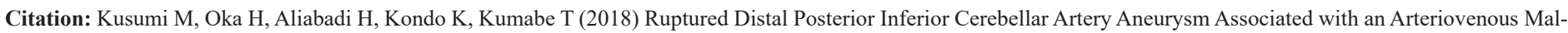

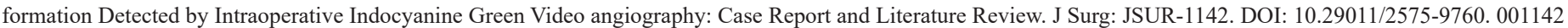

\section{Discussion}

\section{Clinical and Radiological Findings}

The PICA can be divided into five segments named: 1) anterior medullary, 2) lateral medullary, 3) tonsillomedullary, 4) telovelotonsillar, and 5) cortical segments. Distal PICA aneurysms include those arising more peripherally along the vessel without any association with the VA $[3,5,7,8]$. Distal PICA aneurysms are quite rare, up to approximately $1 \%$ of all intracranial aneurysms [4,9]. The majority of patients with distal PICA aneurysms present with hemorrhage and these aneurysms are small in size $[3,4]$. Overall, $63-90 \%$ of previous reported patients experienced bleeding at some time during their clinical course prior to intervention $[3,5,7,9,10]$. Ruptured distal PICA aneurysms result in isolated IVH of the fourth ventricle approximately $50 \%$ of the time $[3,4,11]$. Hokari, et al. reported that all ruptured aneurysms of the choroidal branches of the PICA, except one of eight cases, caused IVH into the fourth ventricle without associated SAH and only one patient presented with IVH with SAH [12]. Vascular anomalies are frequently noted in association with aneurysms of the distal PICA. Regarding previous reports, cerebellar AVMs accompany 4-42\% (mean 23\%) of distal PICA aneurysms, detected by DSA $[5,6,9]$. These AVMs are located at the cerebellar vermis most frequently and next to the cerebellar hemisphere, and are fed by the PICA [3,6,7]. The AVMs usually present with hemorrhage secondary to aneurysmal rupture [7]. Hemodynamic stress-related hyperdynamic flow to the AVMs, has been suggested to be involved in the pathogenesis of these aneurysms $[3,7]$.

One recent paper reported that in five distal PICA aneurysm cases associated with a small cerebellar superior vermian AVM, the AVM nidus was not identified on non-invasive vascular imaging until conventional DSA [6]. At our institution, CTA is the most common initial diagnostic neurovascular tool. Often treatment plans are devised and executed without a DSA, such as in cases of ruptured intracranial aneurysms that are felt to be best secured with open surgical clip ligation. Without DSA, it is quite possible that associated small cerebellar vermian AVMs can go undiagnosed, such as in the case presented here.

\section{Surgical Strategies and Outcome}

The incidence of rebleeding after rupture of distal PICA aneurysms is $78 \%$ and is higher than the incidence of rebleeding in other intracranial aneurysms (which is 34\%) [4,12]. Therefore, ruptured distal PICA aneurysms require immediate management. The management of distal PICA aneurysms is controversial, and various approaches, including direct clipping, trapping with or without distal revascularization, and coiling via the endovascular approach, have been used $[3,6,7,9]$. Combining AVMs have been surgically removed at the time of aneurysmal repair [3] or later by gamma knife [7]. A decision whether to treat the AVM at the same time of aneurysmal surgery should be made, depending on the individual characteristics of the AVM itself, particularly its size, location, and the method chosen to obliterate the aneurysm [7]. In our opinion, direct inspection of the affected segment during open surgery provides several significant advantages compared with endovascular therapy. Direct visualization of the affected arterial segment allows for better decisions regarding the need to spare the parent vessels and to find abnormal vessels $[5,9,13]$. The outcomes of surgically treated patients were generally favorable $[3,7]$.

\section{ICG Video angiography}

Microscopy-integrated ICG video angiography has recently become widespread as an effective method of the surgical treatment of conditions such as AVMs and aneurysms. This imaging technique is useful when disconnecting the abnormal architectures surgically. After ICG injection, which offers realtime information on vessel flow (even with tiny perforators), connecting points of abnormal vessels are clearly identified [14].

In the case illustrated, preoperative CTA was only performed for the detection of the aneurysm and source of hemorrhage, and DSA was not performed. Postoperative DSA revealed the cerebral AVM, and then final diagnosis was made of a ruptured distal PICA aneurysm associated with cerebellar AVM. Intraoperative ICG was found to be very useful in detecting undiagnosed abnormal vessels like AVMs. Thus, we can totally disconnect the abnormal vessel. Finally, this vessel allows us the ability to recognize an associated AVM as well. Most importantly, one needs to be aware that distal PICA aneurysms are often associated with AVMs, possibly undiagnosed by CTA, and DSA should be considered preoperatively to minimize risks of surgery.

\section{Conclusion}

Aneurysms of the distal PICA are associated with isolated fourth ventricular hemorrhages, and are frequently associated with vascular anomalies. Preoperative conventional DSA is required to detect any associated vascular anomalies, even in this era of CTAs. Furthermore, ICG video angiography is a convenient tool to detect such vessel anomalies intraoperatively.

COI Disclosure: The authors have declared no conflicts of interest.

\section{References}

1. Locksley HB (1996) Natural history of subarachnoid hemorrhage, intracranial aneurysms and arteriovenous malformations. Based on 6368 cases in the cooperative study. Journal of neurosurgery 25: 219239

2. Hudgins RJ, Day AL, Quisling RG, Rhoton AL Jr, Sypert GW, et al. (1983) Aneurysms of the posterior inferior cerebellar artery. A clinical 


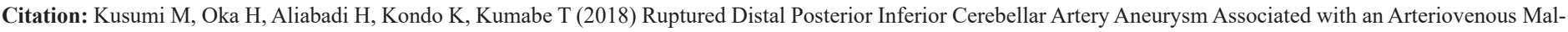

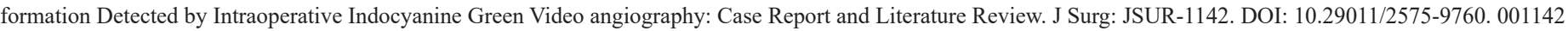

and anatomical analysis. Journal of neurosurgery 58: 381-387.

3. Lewis SB, Chang DJ, Peace DA, Lafrentz PJ, Day AL (2002) Distal posterior inferior cerebellar artery aneurysms: clinical features and management. Journal of neurosurgery 97: 756-766.

4. Ishikawa T, Suzuki A, Yasui N (1990) Distal posterior inferior cerebellar aneurysms--report of 12 cases. Neurologia medico-chirurgica 30: 100-108.

5. Rodriguez-Hernandez A, Zador Z, Rodriguez-Mena R, Lawton MT (2013) Distal aneurysms of intracranial arteries: application of numerical nomenclature, predilection for cerebellar arteries, and results of surgical management. World neurosurgery 80: 103-112.

6. Case D, Kumpe D, Cava L, Neumann R, Roark C, et al. (2016) Ruptured Distal Posterior Inferior Cerebellar Artery (PICA) Aneurysms Associated with Cerebellar Arterial Venous Malformations (AVMs): A Case Series and Review of the Literature Demonstrating the Need for Angiographic Evaluation and Feasibility of Endovascular Treatment. World neurosurgery 2016.

7. Kaptain GJ, Lanzino G, Do HM, Kassell NF (1999) Posterior inferior cerebellar artery aneurysms associated with posterior fossa arteriovenous malformation: report of five cases and literature review. Surgical neurology 51: 146-152.

8. Lister JR, Rhoton AL Jr, Matsushima T, Peace DA (1982) Microsurgical anatomy of the posterior inferior cerebellar artery. Neurosurgery 10: $170-199$
9. Lehto H, Harati A, Niemela M, Dashti R, Laakso A, et al. (2014) Distal posterior inferior cerebellar artery aneurysms: clinical features and outcome of 80 patients. World neurosurgery 82: 702-713.

10. Peluso JP, van Rooij WJ, Sluzewski M, Beute GN, Majoie CB (2008) Posterior inferior cerebellar artery aneurysms: incidence, clinical presentation, and outcome of endovascular treatment. AJNR American journal of neuroradiology 29: 86-90.

11. Tokimura $\mathrm{H}$, Yamahata $\mathrm{H}$, Kamezawa $\mathrm{T}$, Yokoyama $\mathrm{S}$, Haruzono A, et al. (2011) Clinical presentation and treatment of distal posterior inferior cerebellar artery aneurysms. Neurosurgical review 34: 57-67.

12. Hokari M, Kuroda S, Yasuda H, Iwasaki M, Abe S, et al. (2010) Ruptured aneurysms of the choroidal branches of the posterior inferior cerebellar artery: a review of the literature and a case report. Acta neurochirurgica 152: 515-518.

13. Matsushige T, Ikawa F, Ohbyashi N, Imada Y, Kajiwara Y, et al. (2004) [Ruptured aneurysm arising from the anterior medullary segment of the posterior inferior cerebellar artery: report of two cases]. No shinkei geka Neurological surgery 32: 867-874.

14. Sugimoto T, Park YS, Nakagawa I, Nishimura F, Motoyama Y, et al. (2015) Effectiveness of intraoperative indocyanine green video angiography in direct surgical treatment of pediatric intracranial pial arteriovenous fistula. Journal of neurosurgery Pediatrics 15: 55-59. 